\title{
LIPOSARCOMA RETROPERITONEAL GIGANTE
}

\author{
M. PASCUAL SAMANIEGO, J.A. GONZÁLEZ FAJARDO*, F. FERNÁNDEZ DE LA \\ GÁNDARA**, J. CALLEJA ESCUDERO, F.J. SANZ LUCAS, E. FERNÁNDEZ DEL BUSTO \\ Servicio de Urología. *Servicio de Angiología y Cirugía Vascular. ${ }^{* *}$ Servicio de Cirugía General. \\ Hospital Clínico Universitario. Valladolid.
}

Actas Urol Esp. 27 (8): 640-644, 2003

\section{RESUMEN}

LIPOSARCOMA RETROPERITONEAL GIGANTE

El liposarcoma es un tumor maligno de origen mesodérmico derivado del tejido adiposo, que representa la variedad más frecuente dentro de los sarcomas de partes blandas del retroperitoneo aunque globalmente sólo supone el $0,1 \%$ de todas las neoplasias. Su crecimiento es lento y silente siendo su síntoma más frecuente la abdominalgia difusa inespecífica y su signo el aumento del perímetro abdominal. El tratamiento es la cirugía radical siendo básica su resección completa para el control local de la enfermedad. Su tendencia a la afectación multiorgánica y el gran tamaño que alcanzan en su localización retroperitoneal, puede dificultar este objetivo precisando en un alto porcentaje la resección de diferentes órganos de la economía.

Presentamos un caso de liposarcoma retroperitoneal gigante derecho que se manifestó por dolor continuo en el hemiabdomen izquierdo por el desplazamiento visceral, que englobaba el riñón derecho elongando su pedículo vascular y anulando su función.

La alta probabilidad de persistencia de enfermedad microscópica residual obliga a un estricto seguimiento del paciente indicándose en ocasiones el tratamiento complementario con radioterapia.

PALABRAS CLAVE: Tumor retroperitoneal. Liposarcoma. Tratamiento.

\section{ABSTRACT \\ GIANT RETROPERITONEAL LIPOSARCOMA}

Liposarcoma is a malignant tumor that have an embryologic origin from mesodermal tissue depending on fatty tissue. Although liposarcoma is only $0.1 \%$ of all human neoplasms is the most common histology subtype of retroperitoneal soft tissue sarcomas.

This tumor grows slowly. Diffuse abdominal pain is its most frequent symptom and abdominal mass is the most common sign. Aggressive surgical treatment is basic to get a complete resection and a local disease control. This objetive is difficult because of the large tumor size it gets in the retroperitoneal location and the multiorgan involvement that require the resection of a high percentage of contiguous organs.

We report a case of a giant retroperitoneal liposarcoma presenting like continuous left hemiabdominal pain because the visceral compression. The right kidney was involved and suffering from renal vessels enlargement without renal function.

There is a high probability of microscopic residual disease and a good follow-up of the patients is necessary as well as an adjuvant radiation therapy in some cases. 
L os tumores retroperitoneales sólo suponen un 0,07-0,2\%1 de todas las neoplasias del organismo. Un 85\% son malignos, representando los sarcomas de partes blandas el 35\% de este grupo $^{2}$ en el que destaca el liposarcoma como variedad histopatológica más frecuente del retroperitoneo. Este tumor maligno de origen mesodérmico derivado del tejido adiposo, presenta unas características propias en relación con su localización profunda y su crecimiento lento y expansivo, alcanzando diámetros medios de 10 a $15 \mathrm{~cm}$, con compromiso de los órganos vecinos hasta en un $80 \%$ de los $\operatorname{casos}^{3}$. Esto condiciona como signo más frecuente el aumento del perímetro abdominal por una masa palpable. Al ser hipovascular tiene baja tendencia a producir metástasis a distancia dependiendo su pronóstico de su variedad histopatológica y de una posible multicentricidad que dificulte la exéresis quirúrgica radical completa ${ }^{4}$, siendo ésta la única terapia que mejora la supervivencia ${ }^{5}$.

Dada la dificultad para un diagnóstico precoz debido a la inexpresividad clínica del retroperitoneo y a la escasez habitual de sintomas urológicos, parece importante el papel de la exploración abdominal cuidadosa en pacientes con determinados síntomas inicialmente inespecíficos, puesto que la masa abdominal palpable es el dato exploratorio más frecuente ${ }^{6}$, como es el caso de un liposarcoma retroperitoneal gigante que comenzó a manifestarse siete meses antes de su detección.

\section{CASO CLÍNICO}

Mujer de 60 años con antecedentes de hipertensión arterial, paludismo y asma bronquial, que consulta por dolor leve continuo en hipocondrio izquierdo, anorexia, estreñimiento y aumento progresivo del perímetro abdominal con redistribución de la grasa corporal de unos 7 meses de evolución, en ausencia de clínica miccional. A la exploración física presenta distensión abdominal por una gran masa sensible a la palpación, de borde irregular y consistencia firme, que ocupa todo el hemiabdomen derecho. Se aprecia un adelgazamiento en las extremidades, sin edemas ni adenomegalias palpables.

La analítica de sangre y orina es normal. En la radiografía simple de abdomen se aprecia una calcificación de aspecto morular a la derecha de la cuarta vértebra lumbar de unos $8 \mathrm{~cm}$ de diámetro mayor, con desplazamiento del gas intestinal hacia el hemiabdomen izquierdo. La ecografía abdominal informa de una gran masa sólida hiperecogénica y heterogénea que desplaza el riñón derecho hacia la línea media, de límites imprecisos sin poder definir el origen de la misma. El riñón izquierdo es ecográficamente normal.

En la tomografía computerizada (TC), la gran masa intra y retroperitoneal ocupa todo el hemiabdomen derecho sobrepasando la línea media y desplazando anteromedialmente el riñón derecho horizontalizado (Fig. 1). Presenta unos valores de atenuación compatibles con grasa y calcificaciones distróficas en su interior, siendo esto muy sugestivo de liposarcoma.

Se realizó punción aspiración con aguja fina (PAAF) ecodirigida de dicha masa obteniéndose hallazgos histopatológicos sugestivos de liposarcoma.

La resonancia magnética (RM) confirmó el desplazamiento del riñón derecho, el páncreas, las asas intestinales y la vena cava hacia la izquierda por una masa de origen retroperitoneal cuyos septos se realzaban con el contraste intravenoso, con ocupación total del hemiabdomen derecho y ausencia de adenopatías significativas (Figs. 2 y 3).

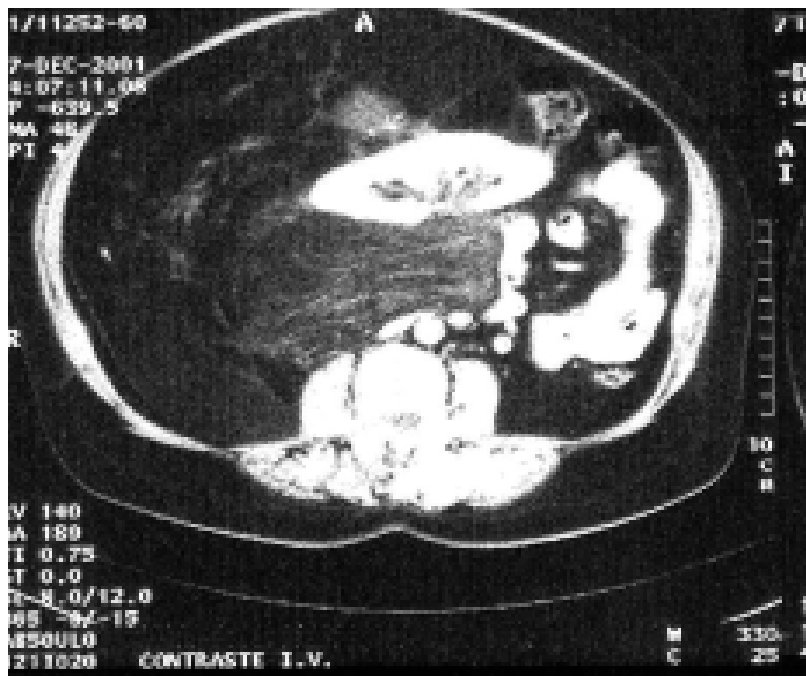

FIGURA 1. Corte abdominal de TC con contraste intravenoso mostrando el desplazamiento y rotación del riñón derecho con elongación de su pedículo vascular. 


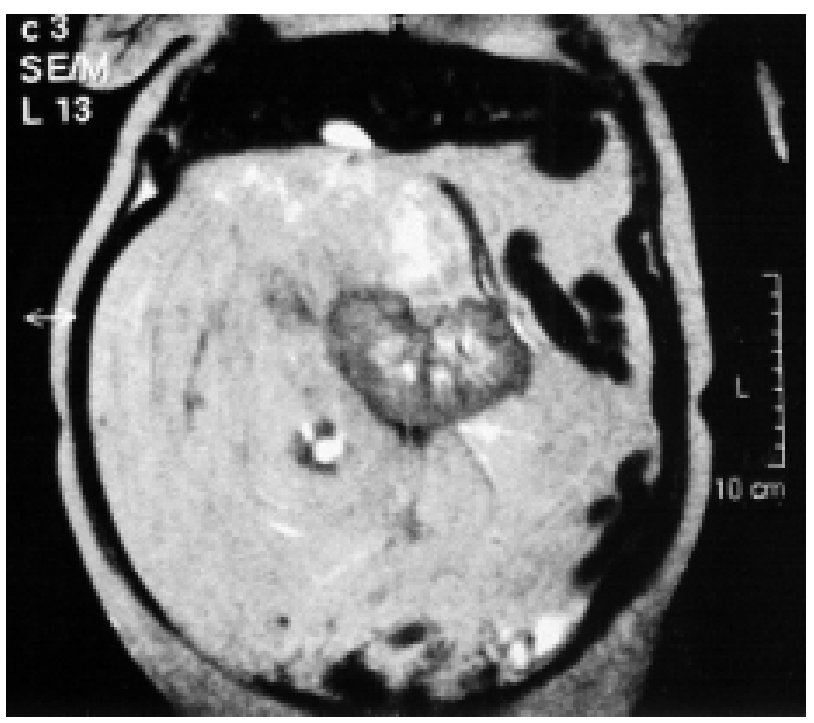

FIGURA 2. Corte frontal de RM: Riñón derecho medializado, horizontalizado $y$ rotado por efecto de una gran masa tumoral que se extiende entre la zona infrahepática y la pelvis sobrepasando la linea media abdominal.

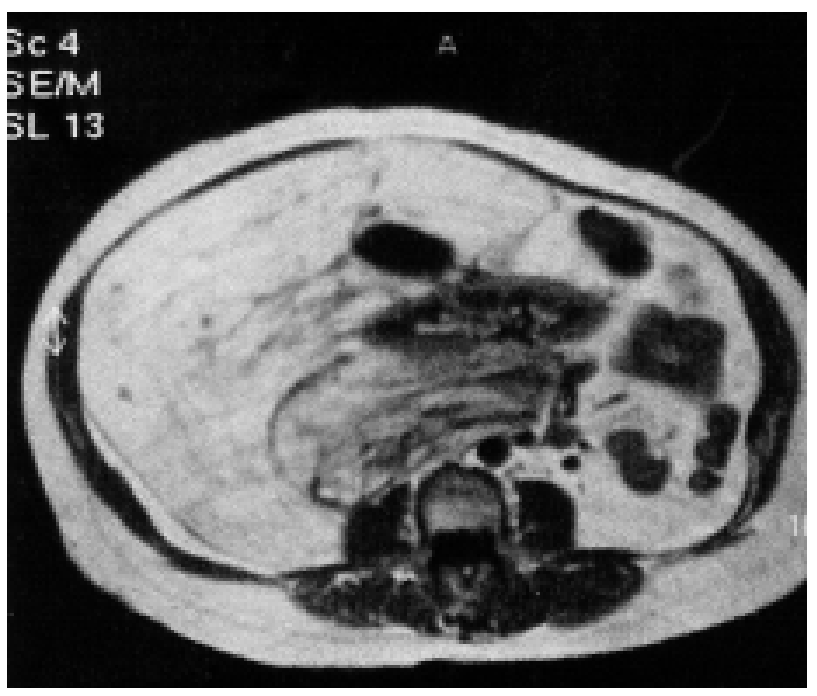

FIGURA 3. Cortes transversales de RM definiendo la extensión del liposarcoma.

La gammagrafía renal con ${ }^{99} \mathrm{Tc}-\mathrm{MDP}$ mostraba ausencia de actividad dependiente del riñón derecho, ausencia de metástasis óseas y captación central de MDP por un posible sarcoma de partes blandas. La radiografía de tórax fue normal.

Tras el estudio preoperatorio se sometió a la paciente a una laparotomía media xifopubiana con disección y exéresis radical de un tumor retroperitoneal bien encapsulado, precisando la realización de nefrectomía derecha ya que la masa envolvía completamente al riñón elongando su pedículo vascular (Fig. 4). El informe anatomopatológico fue de liposarcoma bien diferenciado de 6,5 Kg. de peso que englobaba al riñón derecho disminuido de tamaño y presentando grandes áreas de parénquima fantasma compatibles con infarto renal con calcificación distrófica perirrenal.

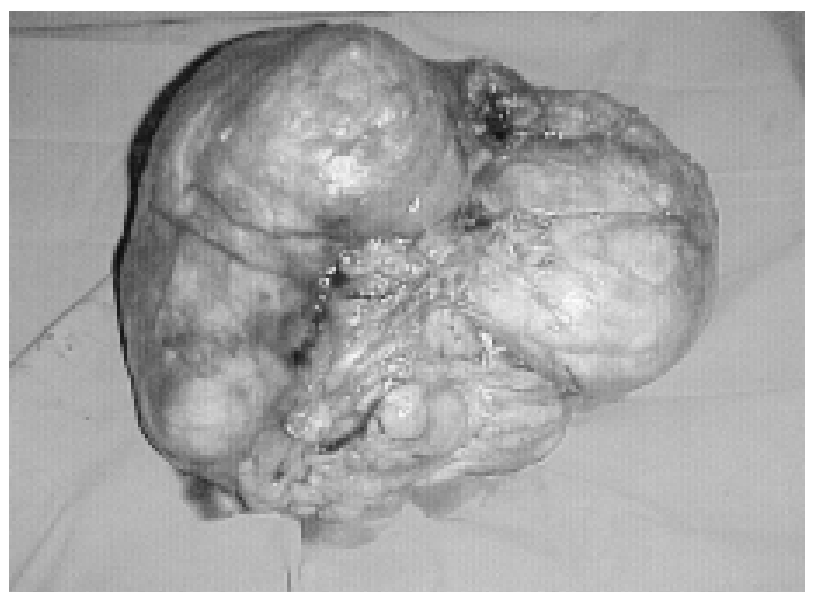

FIGURA 4. Pieza quirúrgica correspondiente a liposarcoma bien diferenciado de $6,5 \mathrm{~kg}$ de peso envolviendo el riñón derecho extirpado con el tumor.

\section{DISCUSIÓN}

Los tumores retroperitoneales son un grupo heterogéneo y poco frecuente de neoplasias, el $85 \%$ malignas $^{7}$, con más de 100 subtipos histológicos $^{8}$ mayoritariamente de origen mesodérmico ${ }^{9}$, caracterizándose por ser tumores hipovasculares, de gran tamaño, con densidad grasa y de etiología desconocida ${ }^{5}$.

Dentro de los sarcomas de partes blandas, el liposarcoma representa de un 7 a $28 \%^{10,11}$ y aunque globalmente sólo supone el 0,1\% de todas las neoplasias del organismo ${ }^{12}$, es considerado el tumor retroperitoneal más frecuente ${ }^{5}$ puesto que el linfoma como enfermedad generalizada no se considera propio del retroperitoneo en sentido estricto ${ }^{8}$.

La localización del liposarcoma en el retroperitoneo es un factor independiente de mal pronóstico asociado a una presentación en la edad adulta (mayores de 50 años), con tamaños tumorales superiores a los $10 \mathrm{~cm}$, con mayor presencia de márgenes quirúrgicos positivos y con afec- 
tación multiorgánica ${ }^{3}$ por su tendencia a la multicentricidad. Esto hace imposible la resección quirúrgica completa hasta en un $83 \%$ de los casos, con porcentajes de reaparición local del tumor entre el 50 y 100\% a partir de tejido residual no extirpado ${ }^{7}$, siendo ésta la principal causa de muerte ${ }^{5}$.

$\mathrm{El}$ otro factor pronóstico es la variedad histopatológica ${ }^{7,13}$, siendo los parámetros más significativos para evaluar el grado tumoral, la diferenciación celular, el número de mitosis y la extensión de la necrosis ${ }^{3}$. Entre los cuatro tipos histológicos de liposarcoma denominados mixoide, pleomórfico, lipoblástico o de células redondas y bien diferenciado o lipoma like ${ }^{14}$, es éste último uno de los más frecuentes y el de mejor pronóstico ${ }^{8}$ por su escasa tendencia a producir metástasis a distancia, aunque muestra una alta incidencia de recurrencia local y cuando esto ocurre suele presentar un fenómeno de desdiferenciación ${ }^{11}$ aumentando su agresividad.

Puede desarrollarse a partir de la grasa perirrenal $^{14}$ e incluso a partir de un lipoma benigno ${ }^{15}$, tratándose de un tumor nodular que por su crecimiento lento y expansivo desarrolla una pseudocápsula formada por células periféricas aplanadas que facilita la disección y exéresis, aunque debido a su tendencia a progresar por los planos aponeuróticos, vasculonerviosos y musculares envolviendo estructuras vitales, es casi imposible conseguir un margen de resección de tejido sano macroscópico ${ }^{8}$. Incluso con márgenes microscópicamente negativos no se puede descartar la existencia de enfermedad microscópica residual, apareciendo recidiva local igualmente que en pacientes con márgenes positivos, por lo que esta circunstancia no supone un factor predictor de recurrencia ${ }^{3}$.

La clínica de consulta suele ser un dolor abdominal difuso ${ }^{5}$ acompañado de un síndrome constitucional propio de una afectación crónica, con anorexia y adelgazamiento de las extremidades por redistribución de la grasa corporal, aumentando el perímetro abdominal ${ }^{8}$. El signo más característico es la masa abdominal palpable indolora detectable en aproximadamente el $78 \%$ de $\operatorname{los} \operatorname{casos}^{9}$, que produce sintomatología abdominal por compresión de órganos, por ejemplo estreñimiento crónico por desplazamiento intestinal como en el caso que presentamos y contras- tando la existencia de una gran masa tumoral con el buen estado general de la paciente como ya han apuntado otros autores ${ }^{13}$.

Nuestra paciente presentó episodios de agudización de su insuficiencia respiratoria asmática fundamentalmente en decúbito, que mejoraron con medidas posturales y oxigenoterapia en relación con una posible compresión diafragmática por el tumor. Esta circunstancia ya ha sido descrita en la literatura ${ }^{8}$.

Aunque el aparato urinario se ve comprometido entre un $72,5 \%$ y un $98,8 \%$ de los casos, la hematuria es excepcional ${ }^{7}$. Nuestra paciente no presentó ningún síntoma genitourinario a pesar del desplazamiento renal derecho más allá de la línea media abdominal, que debido al estiramiento y rotación del hilio renal, acabó infartando el parénquima renal. Esta consecuencia requiere una compresión de gran intensidad por el tumor ${ }^{7}$.

La radiografía simple de abdomen ya aporta datos indirectos de masa retroperitoneal como el desplazamiento de la sombra renal y la existencia de calcificaciones ${ }^{8}$, alcanzando la osificación distrófica en nuestro caso un diámetro de hasta 8 $\mathrm{cm}$. La ecografía facilita datos de localización de una masa sólida pero no permite un diagnóstico diferencial con el angiomiolipoma o grandes tumores renales ${ }^{4}$, e incluso con un aneurisma de aorta o una esplenomegalia ${ }^{13}$.

La TC es una exploración altamente sensible en las neoformaciones retroperitoneales para valorar el tamaño, la extensión y la relación tumoral con los distintos órganos para una correcta planificación quirúrgica y que distingue densidades, sugiriendo la naturaleza del tumor por la hipodensidad característica del tejido adiposo. Sin embargo su resolución puede verse disminuida en presencia de calcificaciones, componentes fibrodensos, necrosis y hemorragia ${ }^{7}$, siendo superada por la RM en cuanto a la diferenciación de tejidos específicos adyacentes y por tanto en el estadiaje tumoral ${ }^{16,17}$. La citología por punción eco o TC dirigida permite filiar la estirpe tumoral de forma preoperatoria.

Dado que la variedad de liposarcoma de bajo grado aparece con más frecuencia a nivel retroperitoneal, es muy importante la exéresis quirúrgica completa que sólo se logra rebasando la cápsula tumoral y realizando en un alto porcentaje de casos la exéresis de órganos englobados por el 
tumor, exigiendo una nefrectomía como en nuestro caso en aproximadamente un $25 \%$ de pacientes $^{5}$. Esto no debe frenar la cirugía dado que la mortalidad operatoria es baja y la enfermedad local persistente es la principal causa de muerte si bien no se ha demostrado una mejora de la supervivencia con la resección de órganos contiguos pero no envueltos por el tumor ${ }^{18}$. Esto exige una exploración minuciosa de todo el lecho abdominal así como una planificación multidisciplinaria del abordaje quirúrgico.

En cuanto a la radioterapia como tratamiento complementario, parece haber acuerdo en su utilización de forma paliativa en tumores no operables o en caso de resección incompleta ${ }^{7,16}$ ya que a pesar de que los tumores mesodérmicos son radiorresistentes, el liposarcoma es el más radiosensible ${ }^{5}$. Aunque se ha apuntado que la radioterapia podría aumentar la supervivencia y el intervalo libre de enfermedad ${ }^{4,7,16}$, hay otros autores para los que este tratamiento no ha demostrado mejorar la supervivencia enfermedad específica a largo plazo en casos de resecciones macroscópicamente completas ${ }^{6,19}$. Esto ocurre incluso a pesar de la utilización de radioterapia intraoperatoria con el fin de aumentar la eficacia de la dosis local con 50-60 $\mathrm{Gy}^{7}$ y de minimizar la toxicidad en los órganos adyacentes, ya que ésta es la principal limitación para la tolerancia a la radioterapia en las estructuras intra-abdominales y retroperitoneales. En nuestro caso, al tratarse de un liposarcoma primario que permitió una resección completa e histopatológicamente bien diferenciado, variedad para la que se han descrito supervivencias a 5 años del $75-100 \%{ }^{16}$, se decidió no aplicar radioterapia ni quimioterapia de acuerdo con la paciente, manteniendo un seguimiento estricto mediante TC o RM cada 6 meses, dado su alto porcentaje de recidivas con un tiempo medio de aparición de 44 meses para estos tumores de bajo grado $^{8}$.

\section{REFERENCIAS}

1. BARREIRAS AF, VICENTE CM, MATEO MA, SUÁREZ MINGUELEZ J.: Tumores del espacio retroperitoneal. A propósito de 7 casos. Rev Quir Esp 1970; 3: 14-25.

2. PACK GT, TABAH EJ.: Primary retroperitoneal tumors. A study of 120 cases. Int Abstr Surg 1954; 99: 209-231.

3. LINEHAN DC, LEWIS JJ, LEUNG D, BRENNAN MF.: Influence of biologic factors and anatomic site in completely resected liposarcoma. J Clin Oncol 2000; 18 (8): 16371643.
4. GUZMÁN MARTÍNEZ-VALLS PL, FERRERO DORIA R, LÓPEZ ALBA J et al.: Liposarcoma retroperitoneal. A propósito de tres casos. Arch Esp de Urol 1997; 50 (5): 529531.

5. AZPIAZU ARNAIZ P, MURO BIDAURRE I, DE FRUTOS GAMERO A et al.: Tumores retroperitoneales. Liposarcoma mixoide retroperitoneal. Presentación de un nuevo caso. Arch Esp de Urol 2000; 53 (2): 170-173.

6. PAREJA IBARS E, ARTIGUES SÁNCHEZ DE ROJAS E, VÁZQUEZ PRADO A, MONTALVÁN ORÓN E, CHECA AYET F, TRULLENQUE PERIS R.: Tumores retroperitoneales: liposarcoma retroperitoneal gigante. Cir Esp 1997; 62: 74-77.

7. ZULUAGA GÓMEZ A, MARTÍNEZ TORRES JL, DE LA FUENTE SERRANO A.: Patología del retroperitoneo. Tumores retroperitoneales. Urolan. Pulso Ediciones 1996; 2: № 1 .

8. VERA DONOSO CD, JIMÉNEZ CRUZ JF.: Tumores retroperitoneales primarios. Tratado de Urología. Tomo II. JR Prous Editores, S.A. 1993: 1323-1336. Barcelona.

9. PASCUAL JI, NAPAL S, SOLCHAGA A.: Diagnóstico por la imagen de los tumores retroperitoneales primitivos. Actas Urol Esp 1991; 15: 154-162.

10. ROSEMBERG S, SUIT H, BAKER L, ROSEN G.: Sarcoma de partes blandas y de los huesos. Principio y prácticas de oncología. Tomo II. Devita V Jr Ed. 1986: 959-1009.

11. EVANS HL.: Liposarcoma: a study of 55 cases with a reassessment of its classification. Am J Surg Pathol 1979; 3: $507-523$

12. BINDER S, KATZ B, SHERIDAN B.: Retroperitoneal liposarcomas. Ann Surg 1979; 187: 257-261.

13. SORIANO F, BAGILET D, CARABALLO E, FEIN L, GUERCETTI R, DIP O.: Liposarcoma retroperitoneal. Análisis clínico patológico de 6 casos. Med Clin 1989; 93: 784785.

14. ENJOJI M, HASHIMOTO H, TSUNEYOSHI M, IWASAKI H.: Malignant fibrous histiocytoma: a clinicopathologic study of 130 cases. Acta Pathol Jpn 1980; 30: 727-741.

15. LE CHEVALIER T, ROUSSÉ J, CONTESSO G, PEJOVIC M, GENIN J, SARRACIN D.: Liposarcomes d'adultes. Elements pronostiques à propos d'une serie de 84 cas. Nouv Presse Med 1981; 10: 3625-3628.

16. ROMERO PÉREZ P, RAFIE MAZKETLI W, AMAT CECILIA M, MERENCIANO CORTINA FJ, GONZÁLEZ DEVESA M.: Tumores adiposos retroperitoneales. A propósito de un liposarcoma mixoide gigante. Actas Urol Esp 1996; 20 (1): 79-84.

17. HRICAK H, DEMAS BE, WILLIAMS RD et al.: Magnetic resonance imaging in the diagnosis and staging of renal and perirrenal neoplasms. Radiology 1985; 154: 709715.

18. BEVILACQUA RG, ROGATKO A, HAJDU SI et al.: Prognostic factors in primary retroperitoneal soft-tissue sarcomas. Arch Surg 1991; 126: 328-334.

19. DÍAZ DEL RÍO M, ABASCAL J, HERRERA N et al.: Tumores retroperitoneales primitivos: experiencia sobre 115 casos. Cir Esp 1991; 49: 129-134.

Dr. M. Pascual Samaniego

C/ Eusebio González Suárez, 3 - 8oD

47014 Valladolid

(Trabajo recibido el 13 noviembre de 2002) 